\title{
Hemagglutinin stalk domain from H5N1 strain as a potentially universal antigen
}

\author{
Karolina Uranowska, Jolanta Tyborowska, Anna Jurek, Bogusław Szewczyk and \\ Beata Gromadzka ${ }^{\bowtie}$ \\ Department of Recombinant Vaccines, Intercollegiate Faculty of Biotechnology, University of Gdansk and Medical University of Gdansk,
} Gdańsk, Poland

Influenza A virus infections are the major public health concern and cause significant morbidity and mortality each year worldwide. Vaccination is the main strategy of influenza epidemic prevention. However, seasonal vaccines induce strain-specific immunity and must be reformulated annually based on prediction of the strains that will circulate in the next season. Thus, it is essential to develop vaccines that would induce broad and persistent immunity to influenza viruses. Hemagglutinin is the major surface antigen of the influenza virus. Recent studies revealed the importance of HA stalk-specific antibodies in neutralization of different influenza virus strains. Therefore, it is important to design an immunogen that would focus the immune response on the HA stalk domain in order to elicit neutralizing antibodies. In the present study, we report characterization of a conserved truncated protein, potentially a universal influenza virus antigen from the H5N1 Highly Pathogenic Avian Influenza A virus strain. Our results indicate that exposure of the HA stalk domain containing conserved epitopes results in cross reactivity with different antibodies (against group 1 and 2 HAs). Additionally, we conclude that HA stalk domain contains not only conformational epitopes recognized by universal FI6 antibody, but also linear epitopes recognized by other antibodies.

Key words: H5N1, universal influenza antigen, hemagglutinin stalk domain, universal influenza antibodies, FI6

Received: 13 June, 2014; revised: 29 August, 2014; accepted: 02 September, 2014; available on-line: 09 September, 2014

\section{INTRODUCTION}

The influenza virus belongs to the Orthomyxoviridae family and is one of the life-threatening pathogens in the world. There are three types of influenza virus identified as $\mathrm{A}, \mathrm{B}$ and $\mathrm{C}$, while subtype $\mathrm{A}$ is predominantly a serious public health problem causing acute infections in humans, characterized by high mortality rate. According to the World Health Organization (WHO), each year influenza virus infects up to $15 \%$ of the population (approximately 1 billion cases). Epidemiological data show a possibility of emergence of new pandemic strains.

Outbreak of the human pandemic influenza A (pH1N1) has caused the intensification of the scientific research and clinical trials so as to develop pandemic influenza vaccines and therapies. Blood samples were collected from donors exposed to pH1N1 and plasma cells were isolated with the aim of screening for secretion of an antibody that would bind to diverse hemagglutinins. A human monoclonal antibody FI6 was found to recognize all subtypes of HAs. FI6 cross-reacts with members of both HAs groups via the attachment to the conserved epitope in hemagglutinin (Corti et al., 2011).

Hemagglutinin (HA) is the major surface glycoprotein of influenza virus. It is a homotrimeric protein molecule, where each monomer consists of two subunits linked by disulfide bonds: HA1 subunit, which forms a globular head and HA2 subunit, which forms a stalk domain (Fig. 1)(Kang et al., 2011). Hemagglutinin plays a crucial role in the viral entry. The globular head domain contains a receptor-binding site for sialic acid that enables attachment of the virus to the host cell, while fusion peptide located in the stalk domain is responsible for the $\mathrm{pH}$-induced fusion of the viral envelope with the endosome membrane (Bouvier \& Palese, 2008).

HA proteins of influenza $A$ viruses are divided into at least 17 subtypes and are categorized into two major phylogenetic groups based on distinct structures in the HA2 stalk domain: group 1 (subtypes H1, H2, H5, H6, H8, H9, H11, H12, H13, H16 and H17) and group 2 (subtypes H3, H4, H7, H10, H14, H15) (Gamblin \& Skehel, 2010; Krammer et al., 2012a).

Current seasonal vaccines predominantly induce anti-HA antibodies that recognize antigenic sites in the globular head domain and act by blocking its receptorbinding activity (Margine et al., 2013a). However, HA head is highly variable among diverse virus strains which is related to frequent amino-acid changes in this region which allows efficient immune evasion. This leads to the lack of protection against viruses that are different from the strain used for vaccine formulation (Margine et al., 2013b).

Recent studies aiming to characterize conserved epitopes of influenza virus revealed that specific regions in the HA stalk domain are highly preserved both in the structure and the sequence among various subtypes of viruses. It has been discovered that neutralizing antibodies to the HA stalk domain can be found after influenza infection (Wrammert et al., 2011) and vaccination (Corti et al., 2010). These anti-stalk antibodies demonstrate cross-reactivity between HAs of many strains from group 1 as well as from group 2 and it has been shown

e-mail: g.beata@biotech.ug.gda.pl

Abbreviations: HA, hemagglutinin; PAb, polyclonal antibody; MAb, monoclonal antibody; HPAl, highly pathogenic avian influenza; $\mathrm{pH} 1 \mathrm{~N} 1$, pandemic strain $\mathrm{H} 1 \mathrm{~N} 1$; $\mathrm{rHA}$, recombinant hemagglutinin; WT AcNPV, Autographa californica nuclear polyhedrosis virus wild type strain. 
A.

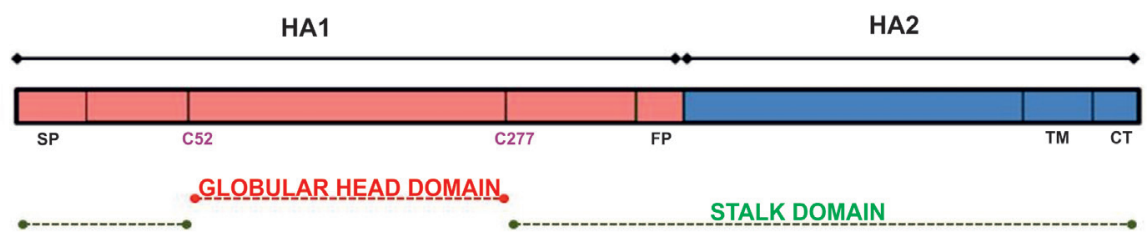

B.

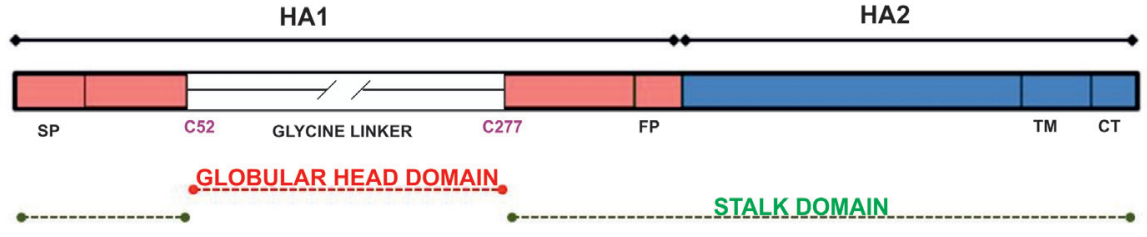

Figure 1. Schematic description of the full length and "headless" hemagglutinin from H5N1 strain.

(A) Full length HA (H5) containing globular head and stalk domain. Globular head region is mainly located between cysteines 52 and 277; stalk domain consists of $\mathrm{N}$ - and C-terminal parts of HA1 subunit and all of HA2 subunit; SP — signal peptide, FP — fusion peptide, TM - transmembrane domain, CT — cytoplasmic tail (B) HA stalk domain construct from the avian influenza virus H5N1 ("headless" HA) lacks the region located between cysteines 52 and 277 on the HA1 subunit and possesses instead a four glycine peptide linker.

that they act by preventing the fusion step of viral entry. It was also confirmed that anti-stalk antibodies elicited by infection with the $2009 \mathrm{pH} 1 \mathrm{~N} 1$ have contributed to the disappearance of normally circulating H1N1 influenza virus strains in the following season (Pica et al., 2012; Sangster et al., 2013).

Antigenically conserved HA stalk domain is thus a promising candidate for preparation of a broadly protective universal influenza vaccine; a potential immunogen that would focus the immune response on the conserved epitopes and would elicit anti-stalk neutralizing antibodies, and may probably give higher protection against different strains of the virus.

In this study we present the design, expression in a baculovirus system and characterization of a conserved truncated protein, HA stalk from the H5N1 HPAI strain, which might potentially be an universal influenza virus antigen. Such a "headless" HA lacks the region located between cysteines 52 and 277 on the HA1 subunit, and instead it posseses a short linker peptide (Fig. 1). Experiments were conducted to determine, whether the antibodies raised against various Influenza A virus strains can bind to HA stalk domain. In addition, we wanted to explore, whether immunization of rabbits with full length H5N1 HA elicits HA stalk-specific antibodies.

\section{MATERIALS AND METHODS}

Virus cDNA synthesis and PCR amplification. The full length HA gene was obtained according to the methods described by Gromadzka et al., 2008. The H5N1 HA stalk domain was designed based on current knowledge of its structure (Steel et al., 2010). Gene coding for the headless hemaglutinin from H5N1 Polish strain was synthesized and cloned into p GEM T-easy vector (accesion number FM163448).

Plasmid construction and sequencing. Products of amplification (full HA and truncated HA) were cloned into pGEM-T easy vector according to the manufacturer's manual (Promega). Plasmids were propagated in Escherichia coli cells and purified on an affinity column (A\&A Biotechnology). DNA sequencing was performed to confirm the correct sequence using universal primers for T7 and Sp6 promoter in pGEM-T easy vector.

Generation of recombinant baculoviruses. Amplified DNA of full-length HA was resolved on 1\% agarose gel, purified (Gel-out, A\&A Biotechnology) and ligated to the pFastBac1 (Invitrogen) donor vector using the BamHI and SpeI restriction enzyme sites. The truncated form of HA gene was cloned into pFastBac1 using the EcoRI and NotI restriction enzyme sites. The resulting recombinant transfer vectors (pFastBac1-HA, pFastBac1HA stalk), which contained full-length HA and truncated form of HA gene respectively, were introduced into the target bacmids by site-specific recombination in E. coli (DH10Bac) as described in the Bac-to-Bac ${ }^{\circledR}$ protocol (Invitrogen).

Cell culture. Spodoptera frugiperda Sf9 insect cells (Invitrogen) were cultured in monolayers at $27^{\circ} \mathrm{C}$ using $\mathrm{HyQ}$ SFX medium (Thermo Scientific). Transfection and recovery of recombinant baculovirus from $S f 9$ cultures was performed according to the Bac-to-Bac ${ }^{\circledR}$ protocol and virus infectivity titer was determined using Sf9-Eeasy Titer (Sf9-ET) cell line. The Sf9-ET cells possess plasmid DNA containing the enhanced green fluorescent protein (eGFP) gene under the control of a baculovirus polyhedrin promoter. When used in the titration assay, the $S f$-9ET cells turn green when they are infected with the baculovirus as a result of the activation of the polyhedrin promoter/eGFP complex by baculovirus gene products expressed during the infection. (Hopkins \& Esposito, 2009). Recombinant baculoviruses containing both forms of HA gene were confirmed by a PCR reaction with specific primers based on a universal sequence in the bacmid genome according to the manual instructions.

Production and purification of hemagglutinin recombinant proteins. Sf9 cells were grown as monolayers at $27^{\circ} \mathrm{C}$ in a 12 -well culture plate using HyQ SFX medium $\left(10^{6}\right.$ cells $\left./ \mathrm{ml}\right)$. Cultures were infected with the recombinant baculovirus with a multiplicity of infection of 1 and cells were collected from the culture medium $48 \mathrm{~h}$ postinfection. Harvested cells and supernatant were separated by centrifugation in a microcentrifuge at 13 $300 \mathrm{rpm}$ for $5 \mathrm{~min}$. Both the supernatant and the cell 
Table 1. Description of different antibodies against group 1 hemagglutinins used in this study.

\begin{tabular}{|c|c|c|c|c|}
\hline \multicolumn{5}{|c|}{ Group 1. Hemagglutinins } \\
\hline & Strain & Immunogen & & Antibody description \\
\hline \multirow[t]{3}{*}{ Human } & $\mathrm{H} 1 \mathrm{~N} 1$ & $\mathrm{HA}$ & $\begin{array}{l}\text { Purified human-cell derived recombinant H1N1 A/ } \\
\text { California/04/2009 and A/California/07/2009 } \\
\text { HA extracellular domain. Clone ID 9G1G8 }\end{array}$ & $\begin{array}{l}\text { Mouse Monoclonal Antibody } \\
\text { Sino Biological Inc. } \\
\text { cat. no } 11048-M M 08\end{array}$ \\
\hline & $\mathrm{H} 1 \mathrm{~N} 1$ & $\mathrm{HA}$ & Purified influenza virus type A strain $\mathrm{H} 1 \mathrm{~N} 1$ & $\begin{array}{l}\text { Mouse Monoclonal Antibody Santa } \\
\text { Cruz Biotechnology } \\
\text { cat. No sc-52025 }\end{array}$ \\
\hline & $\mathrm{H} 1 \mathrm{~N} 1$ & $\mathrm{HA}$ & $\begin{array}{l}\text { Recombinant HA protein eluted from PVDF mem- } \\
\text { brane }\end{array}$ & $\begin{array}{l}\text { Monospecific Rabbit Polyclonal Anti- } \\
\text { body }\end{array}$ \\
\hline \multirow[t]{7}{*}{ Avian/Human } & H5N1 & $\mathrm{HAO}$ & $\begin{array}{l}\text { Recombinant HA protein eluted from PVDF mem- } \\
\text { brane }\end{array}$ & $\begin{array}{l}\text { Monospecific Rabbit Polyclonal Anti- } \\
\text { bodies }\end{array}$ \\
\hline & $\mathrm{H} 5 \mathrm{~N} 1$ & HA1 & 116-256 aa rHA peptide & $\begin{array}{l}\text { Monospecific Rabbit Polyclonal Anti- } \\
\text { bodies }\end{array}$ \\
\hline & $\mathrm{H} 5 \mathrm{~N} 1$ & $\mathrm{HA} 1 / \mathrm{HA} 2$ & $\begin{array}{l}\text { Recombinant HA protein eluted from PVDF mem- } \\
\text { brane }\end{array}$ & $\begin{array}{l}\text { Monospecific Rabbit Polyclonal Anti- } \\
\text { bodies }\end{array}$ \\
\hline & $\mathrm{H} 5 \mathrm{~N} 1$ & $\begin{array}{l}\mathrm{H} 5 \mathrm{~N} 1 \text { - re- } \\
\text { ference }\end{array}$ & Whole virus H5N1: A/Ck/Scot/59 & $\begin{array}{l}\text { Polyclonal Chicken Antibodies - Animal } \\
\text { Health and Veterinary Laboratories } \\
\text { Agency (VLA) } \\
\text { cat. no RAA7002 }\end{array}$ \\
\hline & $\mathrm{H} 5 \mathrm{~N} 1$ & $\mathrm{HA}$ & $\begin{array}{l}\text { Purified human-cell derived recombinant influenza } \\
\text { A virus A/Anhui/1/2005/H5N1 HA extracellular do- } \\
\text { main. Clone ID 9F2E3F3 }\end{array}$ & $\begin{array}{l}\text { Mouse Monoclonal Antibodies. } \\
\text { Sino Biological Inc. } \\
\text { cat. no 11048-MM01 }\end{array}$ \\
\hline & $\mathrm{H} 5 \mathrm{~N} 1$ & $\mathrm{HA}$ & $\begin{array}{l}\text { Purified human-cell derived recombinant influenza } \\
\text { A virus A/Anhui/1/2005/H5N1 HA extracellular do- } \\
\text { main. Clone ID } 1 \text { C5B1A10 }\end{array}$ & $\begin{array}{l}\text { Mouse Monoclonal Antibodies. } \\
\text { Sino Biological Inc. } \\
\text { cat. no } 11048-M M 06\end{array}$ \\
\hline & $\mathrm{H} 5 \mathrm{~N} 1$ & $\mathrm{HA}$ & $\begin{array}{l}\text { Purified human-cell derived recombinant influenza } \\
\text { A virus A/Anhui/1/2005/H5N1 HA extracellular do- } \\
\text { main. Clone ID 14B1E2G6 }\end{array}$ & $\begin{array}{l}\text { Mouse Monoclonal Antibodies. } \\
\text { Sino Biological Inc. } \\
\text { cat. no } 11048-M M 10\end{array}$ \\
\hline \multirow[t]{2}{*}{ Avian } & $\mathrm{H} 5 \mathrm{~N} 2$ & $\begin{array}{l}\mathrm{H} 5 \mathrm{~N} 2-\text { re- } \\
\text { ference }\end{array}$ & Whole virus H5N2:A/Ost/Den/72420/96 & $\begin{array}{l}\text { Chicken Polyclonal Antibody } \\
\text { cat. no RAA7003 1:500 }\end{array}$ \\
\hline & $\mathrm{H} 5 \mathrm{Nx}$ & $\mathrm{H} 5 \mathrm{Nx}$ & Whole virus - H5 avian influenza & $\begin{array}{l}\text { Chicken Polyclonal Antibody } \\
\text { National Veterinary Research Institute } \\
\text { PIWet }\end{array}$ \\
\hline
\end{tabular}

lysates were examined for recombinant proteins (HA full and HA stalk) by SDS-PAGE and Western blotting.

Time course of recombinant $\mathrm{H} 5 \mathrm{~N} 1 \mathrm{HA}$ stalk protein production in Sf9 cells. Sf9 cells were grown as monolayers at $27^{\circ} \mathrm{C}$ in 12 -well culture plate using $\mathrm{HyQ}$ SFX medium $\left(10^{6}\right.$ cells $\left./ \mathrm{ml}\right)$. Cultures were infected with the recombinant baculoviruses at the multiplicity of infection of 1 and cells were collected from the culture medium at different times post infection $(6 \mathrm{~h}, 24 \mathrm{~h}, 48$ h, 72 h, 96 h). Supernatants (medium) were separated from the cell pellet by centrifugation at $13300 \mathrm{rpm}$ for 5 minutes. Proteins from the medium were precipitated using 10\% trichloroacetic acid (TCA). In parallel, cell pellets were treated with lysis buffer on ice $(100 \mathrm{mM}$ Tris $\mathrm{pH} 7.4,100 \mathrm{mM} \mathrm{NaCl}$ and 1\% Nonidet P-40) in order to separate cell membranes from the cytoplasm. Resulting samples were boiled for 10 minutes at $100^{\circ} \mathrm{C}$ in a loading buffer (Life technologies) prior to SDS-PAGE on $10 \%$ gels.

Purification of recombinant HA protein from polyvinylidene difluoride (PVDF) membranes. Cultures were infected with the recombinant baculoviruses at the multiplicity of infection of 1 and cells were collected from the culture medium 72 hours post infection. Supernatants (medium) were separated from the cell pellet by centrifugation at $8500 \mathrm{rpm}$ for 10 minutes. Cell pellets were lysed for $10 \mathrm{~min}$. on ice with a lysis buffer (100 mM Tris pH 7.5, 1\% Triton X-100, $150 \mathrm{mM} \mathrm{NaCl}$ ) in order to separate cell membranes from the cytoplasm. Membrane fraction samples were separated by $8 \%$ SDSPAGE followed by transferring the samples onto PVDF membranes. Electrotransfer was performed in a Tris-glycine transfer buffer at $25 \mathrm{~V}$ overnight. As a control Western Blotting was performed before the blotted proteins were visualized using Ponceau S. HA protein was eluted from PVDF membrane with elution buffer containing 50 $\mathrm{mM}$ Tris $\mathrm{pH} 9.5,2 \%$ SDS and 1\% Triton X-100. Eluted proteins were precipitated with acetone and analyzed by SDS-PAGE gel and Western blot.

Production of hyperimmune antiserum in rabbits. Rabbits were immunized with $100 \mu \mathrm{g}$ of different eluted recombinant $\mathrm{H} 5 \mathrm{~N} 1$ hemmaglutinin (HA0; HA1/HA2) mixed with Freund's complete adjuvant by intradermal injection followed by subcutaneous booster injection 3 weeks later with the same dose in Freund's incomplete adjuvant. Rabbits were bled 2 weeks later. The serum was collected and stored at $-20^{\circ} \mathrm{C}$ until use.

Immunoperoxidase Monolayer Assay (IPMA). Sfg cells were seeded into 12 -well cell culture plates $\left(10^{6}\right.$ cells $/ \mathrm{ml}$ ) and infected with the recombinant baculoviruses at the multiplicity of infection of 1 . After incubating for 48 hours, the medium was removed and the cells 
Table 2. Description of different antibodies against group 2 hemagglutinins used in this study.

\begin{tabular}{|c|c|c|c|c|}
\hline \multicolumn{5}{|c|}{ Group 2. Hemagglutinins } \\
\hline & Strain & Immunogen & & Antibody description \\
\hline Human & $\mathrm{H} 3 \mathrm{~N} 2$ & $\mathrm{HA}$ & $\begin{array}{l}\text { Purified human-cell derived recombinant } \\
\text { H3N2 A/Brisbane/10/2007 HA. } \\
\text { Clone ID MM03 }\end{array}$ & $\begin{array}{l}\text { Mouse Monoclonal Antibody } \\
\text { Sino Biological Inc. } \\
\text { cat. no 11056-MM03 }\end{array}$ \\
\hline Avian/Human & H7N9 & $\mathrm{HA}$ & $\begin{array}{l}\text { Purified recombinant Influenza A } \\
\text { H7N9/A/Shanghai/1/2013 } \\
\text { HA } 1-524 \text { aa }\end{array}$ & $\begin{array}{l}\text { Rabbit Polyclonal Antibody } \\
\text { Sino Biological Inc. } \\
\text { cat. no 40104-RP02 }\end{array}$ \\
\hline Avian/Human & H7N9 & $\mathrm{HA}$ & $\begin{array}{l}\text { Purified recombinant Influenza A H7N9 } \\
\text { /A/Shanghai/1/2013 } \\
\text { HA } 1-524 \text { aa }\end{array}$ & $\begin{array}{l}\text { Mouse Monoclonal Antibody } \\
\text { Sino Biological Inc. } \\
\text { cat. no 11082-MM04 }\end{array}$ \\
\hline Avian & $\mathrm{H} 7 \mathrm{Nx}$ & $\begin{array}{l}\text { H7Nx avian } \\
\text { influenza }\end{array}$ & Whole virus- H7/Poland & $\begin{array}{l}\text { Chicken Polyclonal Antibody } \\
\text { National Veterinary Research Institute PIWet }\end{array}$ \\
\hline Avian & H4N6 & $\mathrm{HA}$ & $\begin{array}{l}\text { Purified recombinant Influenza A } \\
\text { H4N6/A/mallard/Ohio/657/2002 } \\
\text { HA } 1-524 \text { aa }\end{array}$ & $\begin{array}{l}\text { Rabbit Polyclonal Antibody } \\
\text { Sino Biological Inc. } \\
\text { Cat.no 11714-RP01 }\end{array}$ \\
\hline Human & Fl6 & $\mathrm{HA}$ & H1N1 v & $\begin{array}{l}\text { Highly specific humanised synthetic antibodies } \\
\text { based on neutralizing antibody selected from } \\
\text { plasma cells that bind to group } 1 \text { and group } 2 \\
\text { Influenza A HAs }\end{array}$ \\
\hline
\end{tabular}

were fixed with $70 \%$ methanol. Cells were then incubated with primary antibodies (Tables 1-4) in a binding buffer (PBS - 0.05\% Tween 80, 5\% FBS) for $1 \mathrm{~h}$, at RT. Cells were then washed three times with PBS - 0.05\% Tween 80, horseradish peroxidase conjugated secondary antibody (Santa Cruz Biotechnology, USA) was added at a dilution 1:1000 and plates were incubated for $1 \mathrm{~h}$, at RT. After a final wash, NovaRED Peroxidase Substrate Kit (Vector) was used in order to detect positive red reaction products. The color reaction was allowed to develop for 5-15 minutes and the plates were then washed with $\mathrm{H}_{2} \mathrm{O}$ and examined under a light microscope.

Western blot analysis. Proteins were separated by $10 \%$ SDS-PAGE followed by samples transfer to PVDF membranes. After protein electrotransfer in a Tris-glycine buffer at $25 \mathrm{~V}$ overnight, membranes were blocked with $3 \%$ non-fat milk at RT for $1 \mathrm{~h}$ and incubated with different antibodies against HA proteins (Tables 1-4) at RT for $1 \mathrm{~h}$. Subsequently, alkaline phosphatase-conjugated secondary antibody (1:1000, Santa Cruz Biotechnology, USA) was added and it was incubated for $1 \mathrm{~h}$ at RT. After three washes with TBS, a substrate solution containing NBT and BCIP was added. After developing the bands, the membrane was washed with water.

Flow cytometry. Sf9 cells were seeded into 6-well cell culture plates $\left(1.5 \times 10^{6}\right.$ cells $\left./ \mathrm{ml}\right)$ and infected with the recombinant baculoviruses at the multiplicity of infection of 1. Presence of full and stalk recombinant HA proteins at the cell surface was determined by indirect immunofluorescence as follows. Cells $\left(5 \times 10^{5}\right.$ cells $/$ sample $)$ were collected into $100 \mu \mathrm{l}$ of a PBA buffer (PBS $+1 \%$ $\mathrm{BSA}+0.02 \%$ sodium azide) and placed separately into the wells of 96-well polystyrene V-bottom microplate (Greiner Bio-One, Germany). Next, the cells were centrifuged for $3 \mathrm{~min}$ at $1300 \mathrm{rpm}$.

Cells were resuspended in the primary antibody solution in PBA (Tables 3-4) and stained for 1 hour on ice. After two rounds of washing with PBA by 3 min centrifugation at $1300 \mathrm{rpm}, 4^{\circ} \mathrm{C}$, discarding the antibody solution and resuspending the cell pellet in the PBA washing buffer, cells were incubated with goat anti-mouse IgG-PE (1:1000, Becton-Dickinson, USA) for $45 \mathrm{~min}$ on ice. After double washing with PBA, cells were re- suspended in $120 \mu \mathrm{l}$ of PBA and transferred to $0.65 \mathrm{ml}$ tubes. Cells were analyzed using the FACS Calibur flow cytometer (Becton-Dickinson, USA) and the CellQuest software (Becton-Dickinson, USA).

Antibodies recognizing various hemagglutinins. Different monospecific rabbit polyclonal antibodies (anti HA0 H5N1, anti HA1/HA2 H5N1) were obtained in our laboratory (unpublished data). Chicken polyclonal antibodies (anti $\mathrm{H} 5 \mathrm{Nx}, \mathrm{H} 1 \mathrm{~N} 1, \mathrm{H} 7 \mathrm{Nx}$ ) were kindly provided by National Veterinary Research Institute (PIWet). The rest of the used antibodies were purchased from Santa Cruz Biotechnology Inc., Sino Biological Inc. and Veterinary Laboratories Agency (VLA). The details are presented in Tables 1 and 2 .

\section{RESULTS}

\section{Expression of the hemagglutinin recombinant protein genes in Sf9 insect cells}

Our aim was to investigate whether the approach based on presentation to the host immune system of a stalk region of hemagglutinin of the human influenza viruses (H1 and H3) described by Steel and coworkers, 2010 can be applied also to the HPAI strains. For this purpose we planned to characterize the conserved, truncated form of hemagglutinin (HA stalk) from the Polish H5N1 strain. To compare the results obtained for truncated form of HA we decided to express the full length HA gene as well. Recombinant viruses bearing the full length and stalk form of hemagglutinin were used to infect insect cells under optimized conditions. A major polypeptide band of full-length HA0 with a molecular mass of $70 \mathrm{kDa}$ was identified in cell lysates by SDSPAGE followed by either Coomassie brilliant blue staining or Western blot analysis using monoclonal antibodies anti-HA H5N1 (1:1000, Sino Biological Inc.) (Fig. 2).

All the HPAI hemagglutinins possess multiple arginine residues at the site of cleavage and are cut intracellulary by ubiquitously occurring proteases (Steinhauer, 1999; Gamblin \& Skehel, 2010). Apart from recombinant HA0 we could also observe HA1 with molecular mass of 53 $\mathrm{kDa}$ and HA2 subunits of $30 \mathrm{kDa}$ which were the re- 
Table 3. Reactivity of H5N1 HA stalk recombinant protein with antibodies against group 1 hemagglutinins.

\begin{tabular}{|c|c|c|c|c|c|c|c|c|c|c|c|c|c|c|}
\hline \multirow{2}{*}{\multicolumn{3}{|c|}{ Group 1. Hemagglutinins }} & \multirow{2}{*}{\multicolumn{4}{|c|}{$\begin{array}{l}\text { IPMA } \\
\text { H5N1 antigen }\end{array}$}} & \multirow{2}{*}{\multicolumn{4}{|c|}{$\begin{array}{l}\text { Western Blotting } \\
\text { H5N1 antigen }\end{array}$}} & \multirow{2}{*}{\multicolumn{4}{|c|}{$\begin{array}{l}\text { FACS } \\
\text { H5N1 antigen }\end{array}$}} \\
\hline & & & & & & & & & & & & & & \\
\hline & & ANTIBODY & $\begin{array}{l}\text { Bac } \\
\text { HA } \\
\text { stalk }\end{array}$ & $\begin{array}{l}\text { Bac } \\
\mathrm{HA} \\
\text { full }\end{array}$ & $\begin{array}{l}\mathrm{Bac} \\
\mathrm{WT}\end{array}$ & $\begin{array}{l}\text { Mock } \\
\text { (Sf9) }\end{array}$ & $\begin{array}{l}\text { Bac } \\
\text { HA } \\
\text { stalk }\end{array}$ & $\begin{array}{l}\text { Bac } \\
\text { HA } \\
\text { full }\end{array}$ & $\begin{array}{l}\text { Bac } \\
\text { WT }\end{array}$ & $\begin{array}{l}\text { Mock } \\
\text { (Sf9) }\end{array}$ & $\begin{array}{l}\text { Bac } \\
\mathrm{HA} \\
\text { stalk }\end{array}$ & $\begin{array}{l}\text { Bac } \\
\text { HA } \\
\text { full }\end{array}$ & $\begin{array}{l}\text { Bac } \\
\text { WT }\end{array}$ & $\begin{array}{l}\text { Mock } \\
\text { (Sf9) }\end{array}$ \\
\hline H1N1 & anty $\mathrm{HA}$ & $\begin{array}{l}\text { Mouse MAb anty } \\
\text { HA H1N1 } \\
\text { Sino Biological Inc. } \\
\text { 1:1000 }\end{array}$ & +++ & - & - & NT & +++ & NT & - & - & ++ & - & - & - \\
\hline $\mathrm{H} 1 \mathrm{~N} 1$ & anty $\mathrm{HA}$ & $\begin{array}{l}\text { Mouse MAb anty } \\
\text { HA H1N1 Santa } \\
\text { Cruz Biotech. } \\
\text { 1:1000 }\end{array}$ & +++ & - & - & NT & +++ & - & - & - & +++ & + & - & - \\
\hline H1N1 & anty $\mathrm{HA}$ & $\begin{array}{l}\text { Monospecific Rab- } \\
\text { bit PAb } \\
\text { 1:500 }\end{array}$ & +++ & + & - & NT & +++ & NT & - & - & NT & NT & NT & NT \\
\hline $\mathrm{H} 1 \mathrm{~N} 1$ & $\begin{array}{l}\text { anty } \\
\text { H1N1 } \\
\text { avian in- } \\
\text { fluenza }\end{array}$ & $\begin{array}{l}\text { Chicken PAb } \\
\text { National Veterinary } \\
\text { Research Institute } \\
\text { PIWet 1:500 }\end{array}$ & NT & NT & NT & NT & ++ & ++ & - & - & NT & NT & NT & NT \\
\hline H5N1 & $\begin{array}{l}\text { anty } \\
\text { HAO }\end{array}$ & $\begin{array}{l}\text { Monospecific Rab- } \\
\text { bit PAb } \\
1: 1000\end{array}$ & +++ & +++ & + & NT & +++ & +++ & + & - & NT & NT & NT & NT \\
\hline H5N1 & $\begin{array}{l}\text { anty } \\
\mathrm{HA} 1\end{array}$ & $\begin{array}{l}\text { Monospecific Rab- } \\
\text { bit PAb } \\
1: 1000\end{array}$ & - & +++ & - & NT & & & & & NT & NT & NT & NT \\
\hline H5N1 & $\begin{array}{l}\text { anty } \\
\text { HA1/ } \\
\text { HA2 }\end{array}$ & $\begin{array}{l}\text { Monospecific Rab- } \\
\text { bit PAb } \\
\text { 1:500 }\end{array}$ & +++ & +++ & + & NT & +++ & +++ & + & NT & NT & NT & NT & $\mathrm{NT}$ \\
\hline H5N1 & $\begin{array}{l}\text { anty } \\
\text { H5N1 } \\
\text { - refe- } \\
\text { rence }\end{array}$ & $\begin{array}{l}\text { Chicken PAb VLA } \\
1: 1000\end{array}$ & +++ & +++ & - & NT & +++ & +++ & - & NT & NT & NT & NT & NT \\
\hline H5N1 & anty $\mathrm{HA}$ & $\begin{array}{l}\text { Mouse MAb anty } \\
\text { HA H5N1 } \\
\# 11048-M M 01 \\
\text { Sino Biological Inc. } \\
\text { 1:1000 }\end{array}$ & + & - & - & - & +++ & - & - & - & - & + & - & - \\
\hline H5N1 & anty $\mathrm{HA}$ & $\begin{array}{l}\text { Mouse MAb anty } \\
\text { HA H5N1 } \\
\# 11048-M M 06 \\
\text { Sino Biological Inc. } \\
\text { 1:1000 }\end{array}$ & +++ & - & - & - & NT & +++ & - & - & NT & NT & NT & NT \\
\hline H5N1 & anty HA & $\begin{array}{l}\text { Mouse MAb anty } \\
\text { HA H5N1 } \\
\# 11048-M M 10 \\
\text { Sino Biological Inc. } \\
\text { 1:1000 }\end{array}$ & - & - & - & - & NT & - & - & - & NT & NT & NT & NT \\
\hline $\mathrm{H} 5 \mathrm{~N} 2$ & $\begin{array}{l}\text { anty } \\
\mathrm{H} 5 \mathrm{~N} 2 \\
\text { - refe- } \\
\text { rence }\end{array}$ & $\begin{array}{l}\text { Chicken PAb VLA } \\
\text { \# RAA7003 } \\
\text { 1:500 }\end{array}$ & +++ & +++ & - & NT & ++ & +++ & - & - & NT & NT & NT & NT \\
\hline $\mathrm{H} 5 \mathrm{Nx}$ & $\begin{array}{l}\text { anty } \\
\text { H5Nx }\end{array}$ & $\begin{array}{l}\text { Chicken PAb } \\
\text { National Veterinary } \\
\text { Research Institute } \\
\text { PIWet 1:500 }\end{array}$ & + & + & - & NT & +++ & ++ & - & - & NT & $\mathrm{NT}$ & NT & NT \\
\hline
\end{tabular}

NT, not tested; -, negative reaction; +++ , the strongest positive reaction; + , the weakest positive reaction

sult of a proteolytic cleavage by insect cells protease furin (Fig. 2b). The recombinant HA stalk protein was also detected using anti-HA0 H5N1 rabbit polyclonal serum (1:800, obtained in our laboratory). We detected HA stalk protein mainly in the membranes which is similar to cell localization of full length HA (Fig. 3). In western blot analysis under reducing conditions we observed three major bands of different forms of recombinant HA stalk protein. The observed three forms of expressed recombinant protein most likely represent: HA stalk composed of $\mathrm{N}$ - and C-terminal parts of HA1 subunit plus the whole HA2 subunit with molecular mass of $45 \mathrm{kDa}$, single HA2 subunit $(37 \mathrm{kDa})$ and $\mathrm{N}$ - and C-terminal parts of HA1 subunit linked by four glycines $(27 \mathrm{kDa})$.

We wanted to investigate whether absence of the globular head domain would disrupt transport through the Golgi complex to the cell surface. We performed 
Table 4. Reactivity of H5N1 HA stalk recombinant protein with antibodies against group 2 hemagglutinins.

\begin{tabular}{|c|c|c|c|c|c|c|c|c|c|c|c|c|c|c|}
\hline \multirow{2}{*}{\multicolumn{3}{|c|}{ Group 2. Hemagglutinins }} & \multicolumn{4}{|l|}{ IPMA } & \multicolumn{4}{|c|}{ Western Blotting } & \multicolumn{4}{|c|}{ FACS } \\
\hline & & & \multicolumn{4}{|c|}{ H5N1 antigen } & \multicolumn{4}{|c|}{ H5N1 antigen } & \multicolumn{4}{|c|}{ H5N1 antigen } \\
\hline & & Antibody & $\begin{array}{l}\text { Bac } \\
\text { HA } \\
\text { stalk }\end{array}$ & $\begin{array}{l}\text { Bac } \\
\text { HA } \\
\text { full }\end{array}$ & $\begin{array}{l}\text { Bac } \\
\text { WT }\end{array}$ & $\begin{array}{l}\text { Mock } \\
\text { (Sf9) }\end{array}$ & $\begin{array}{l}\text { Bac } \\
\text { HA } \\
\text { stalk }\end{array}$ & $\begin{array}{l}\text { Bac } \\
\text { HA } \\
\text { full }\end{array}$ & $\begin{array}{l}\text { Bac } \\
\text { WT }\end{array}$ & $\begin{array}{l}\text { Mock } \\
\text { (Sf9) }\end{array}$ & $\begin{array}{l}\text { Bac } \\
\text { HA } \\
\text { stalk }\end{array}$ & $\begin{array}{l}\text { Bac } \\
\text { HA } \\
\text { full }\end{array}$ & $\begin{array}{l}\text { Bac } \\
\text { WT }\end{array}$ & $\begin{array}{l}\text { Mock } \\
\text { (Sf9) }\end{array}$ \\
\hline $\mathrm{H} 3 \mathrm{~N} 2$ & anty $\mathrm{HA}$ & $\begin{array}{l}\text { Mouse MAb } \\
\text { Sino Biological Inc. } \\
\# 11056-M M 03 \\
\text { 1:1000 }\end{array}$ & - & - & - & - & NT & NT & NT & NT & + & - & - & - \\
\hline H7N9 & anty $\mathrm{HA}$ & $\begin{array}{l}\text { Rabbit PAb } \\
\text { Sino Biological Inc. } \\
\text { \#40104-RP02 } \\
\text { 1:1000 }\end{array}$ & - & - & - & - & NT & NT & NT & NT & NT & NT & NT & NT \\
\hline H7N9 & anty $\mathrm{HA}$ & $\begin{array}{l}\text { Mouse MAb } \\
\text { Sino Biological Inc. } \\
\# 11082-M M 04 \\
\text { 1:1000 }\end{array}$ & - & - & - & - & NT & NT & NT & NT & ++ & - & - & - \\
\hline H7NX & $\begin{array}{l}\text { anty } \\
\text { H7Nx } \\
\text { avian } \\
\text { influenza }\end{array}$ & $\begin{array}{l}\text { Chicken PAb } \\
\text { National Veterinary } \\
\text { Research Institute } \\
\text { PIWet 1:500 }\end{array}$ & ++ & + & - & NT & + & - & - & - & NT & NT & NT & NT \\
\hline H4N6 & anty $\mathrm{HA}$ & $\begin{array}{l}\text { Rabbit PAb } \\
\text { Sino Biological Inc. } \\
\# 11714-R P 01 \\
\text { 1:1000 }\end{array}$ & ++ & - & - & - & NT & NT & NT & NT & NT & NT & NT & NT \\
\hline
\end{tabular}

NT, not tested; -, negative reaction; +++ , the strongest positive reaction; + , the weakest positive reaction

fluorescence-activated cell sorter analysis of infected insect cells with recombinant baculoviruses (Bac HA, Bac HA stalk) following surface staining with HA specific antibodies (Table 3). We could detect the homotrimeric form of HA stalk protein $(130 \mathrm{kDa})$ in the membrane fraction (Fig. 4a) and on the surface of the infected cells (Fig. 4c), which indicates that disulfide bond formation between Cys 52 and 277 is probably conserved among all avian and human influenza viruses.

\section{Production of hyperimmune antiserum in rabbits}

Analyses of the human antibody repertoire after infection with pandemic H1N1 strain, revealed presence of

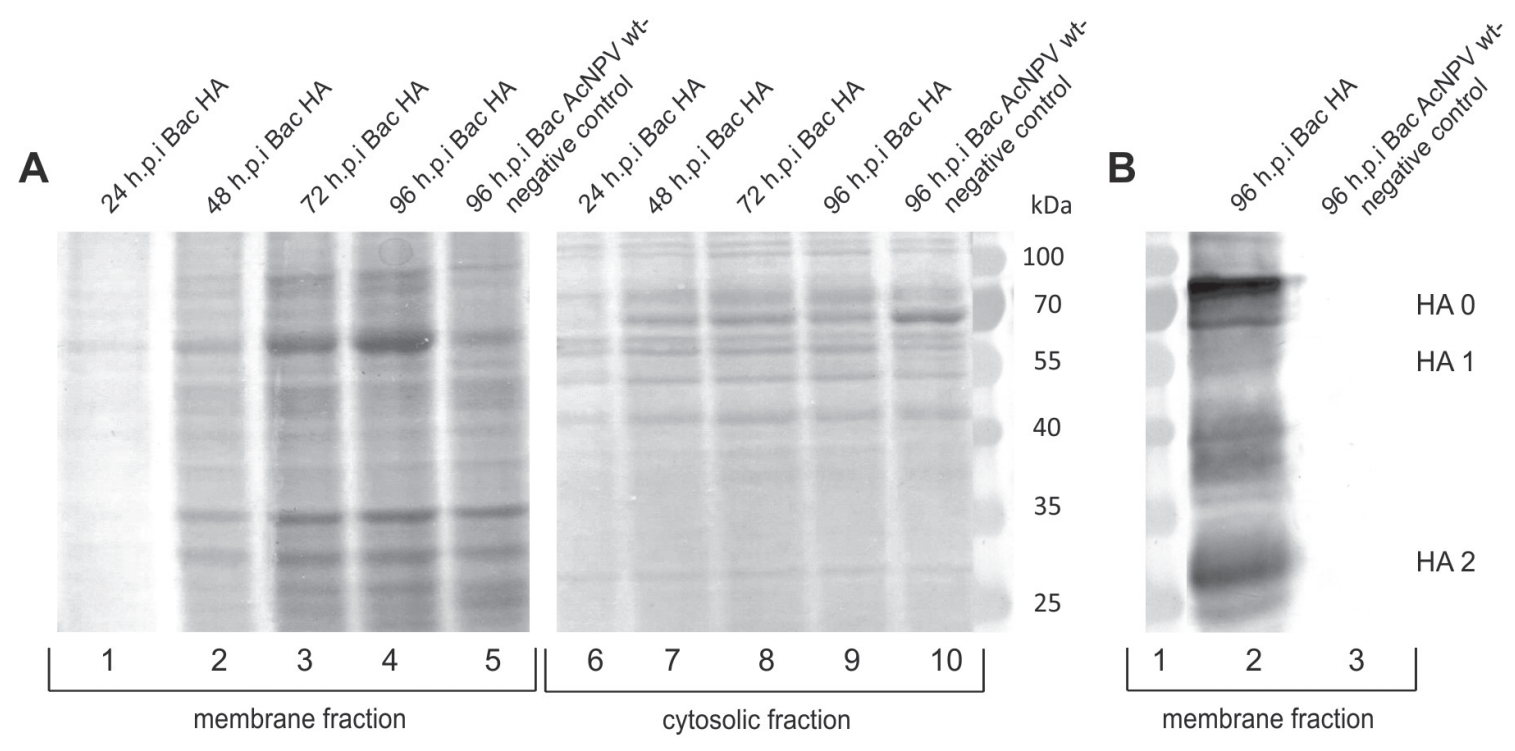

Figure 2. Analysis of the time course of protein production of Influenza Virus H5N1 full length hemagglutinin in insect cells .

Cell membrane fraction and cytoplasm were examined by SDS-PAGE under reducing conditions $\left(0.5 \times 10^{6}\right.$ cells/well). The fractions were collected in 24, 48, 72 or 96 hours post infection. (A) 10\% SDS-PAGE gel stained with Coomassie blue-R 250. Lane 1, 2, 3, 4, 5 insoluble fraction; Lane 6, 7, 8, 9, 10, soluble fraction. (B) Western blot analysis of rHA H5N1 using monoclonal antibodies anti-HA H5N1 (1:1000 Sino Biological Inc.) HAO - $70 \mathrm{kDa}$; HA1 - $53 \mathrm{kDa}$; HA2 - $30 \mathrm{kDa}$; Lane 1 Protein Ladder — PageRuler Prestained Protein Ladder (Fermentas); Lane 2 insoluble fraction; Lane 3 insoluble fraction of negative control. 

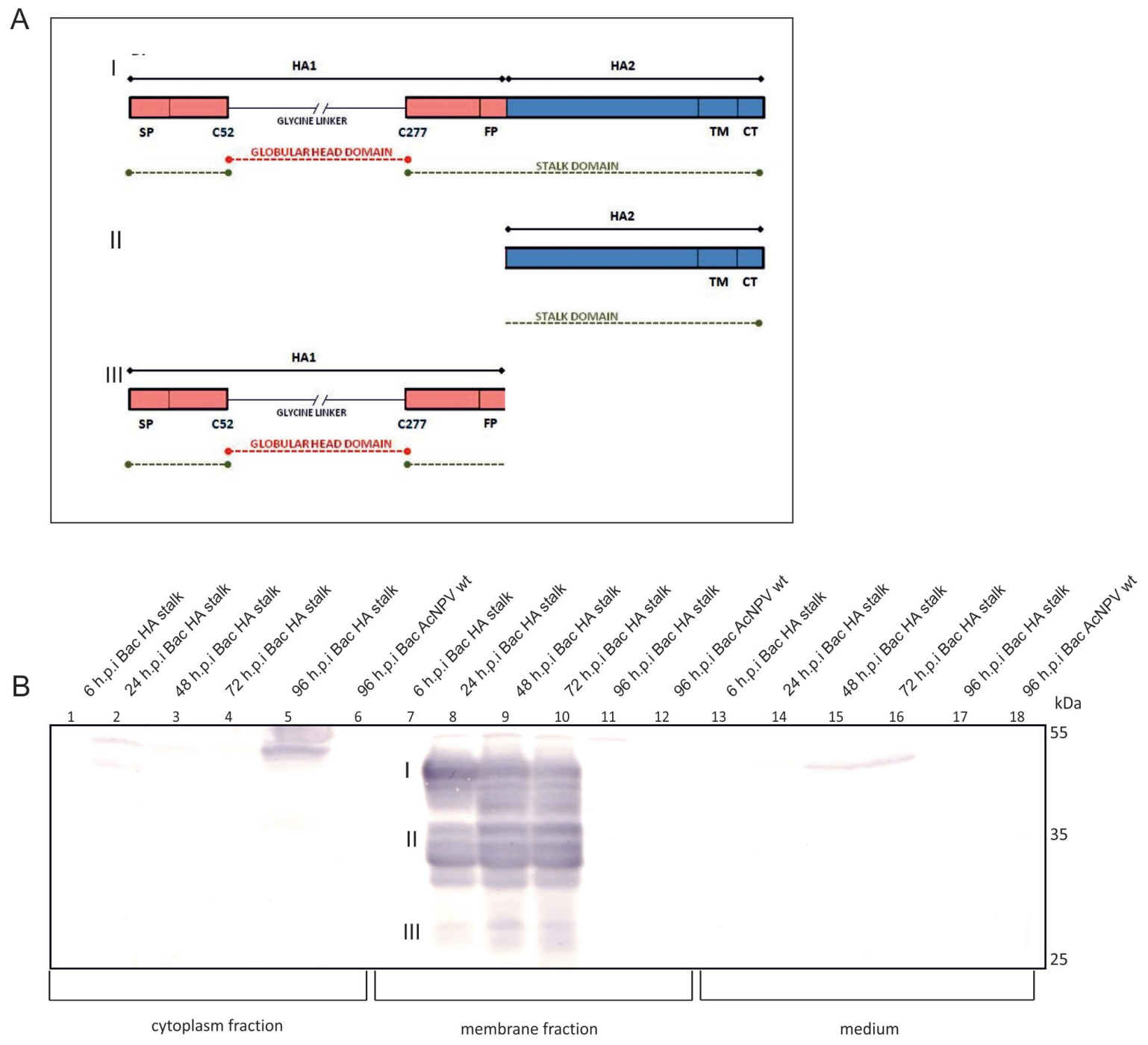

Figure 3. Analysis of the production time course of Influenza Virus H5N1 hemagglutinin stalk domain in insect cells.

Cell membrane fraction, cytoplasm and medium were examined by SDS-PAGE under reducing conditions ( $0.5 \times 10^{6}$ cells/lane). Fractions were collected in 6, 24, 48, 72 or 96 hours post infection. (A) Schematic description of three different forms of HA stalk protein. (B) Western Blot analysis of the hemagglutinin stalk domain expressed in Sf9 cells was performed. Anti-HAO H5N1 rabbit polyclonal serum (1:800 dilution; anti-HA0 H5N1) was used to detect the recombinant protein - HA stalk.

broadly neutralizing antibodies against influenza hemagglutinin.

With the purpose of investigation whether the vaccination with full length recombinant hemagglutinin from H5N1 strain elicits similar antibodies recognizing the conserved epitopes localized in the HA stalk domain we decided to examine sera that were obtained earlier (unpublished data).

Rabbits were immunized with recombinant full-length HA obtained in different forms: uncleaved HA0 protein and two subunits HA1/HA2 together. The sera obtained had high titers of antibodies against full length HA when tested in Western blotting, comparable to the ones commercially available. (Fig. 5). As shown in Fig. 3, the antiHA0 H5N1 serum reacted with linear epitopes localized in the conserved HA stalk region (HA2 subunit). Positive results were also obtained for conformational antigen - HA stalk in IPMA test (Fig. 6).

As many scientist described the cross reactivity of antibodies raised against a pandemic recombinant he- magglutinin from H1N1 strain with different HAs, here we report for the first time the reactivity of antibodies to HA0 H5N1 with conserved region of HA2 subunit (H5N1 HA stalk). We suppose that specific structure of pandemic HA H1N1 triggers the production of antibodies recognizing conserved epitopes but in this study we present that the HA H5N1 also stimulates production of antibodies that recognized universal epitopes (Table 3).

\section{Reactivity of HA stalk with different antibodies}

Expression of the recombinant H5N1 HA stalk protein was confirmed by SDS-PAGE and Western blot as described above. Recombinant protein was characterized by its reactivity with various antibodies in the IPMA test, confirmed by Western blotting and FACS (Tables 1-4). Exposure of the HA stalk domain from the avian influenza virus $\mathrm{H} 5 \mathrm{~N} 1$ strain resulted in higher reactivity with different antibodies directed against various hemagglutinins. 
A

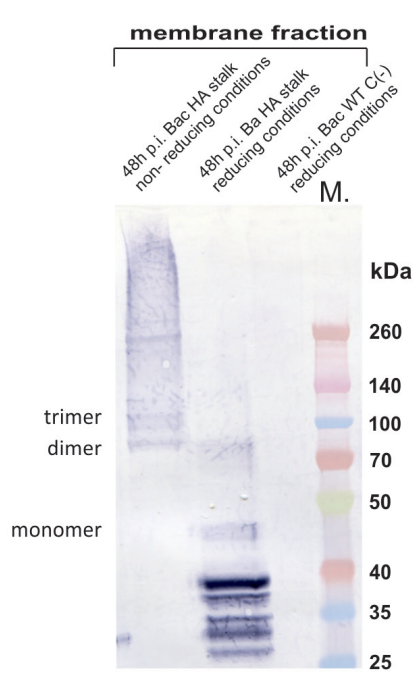

B

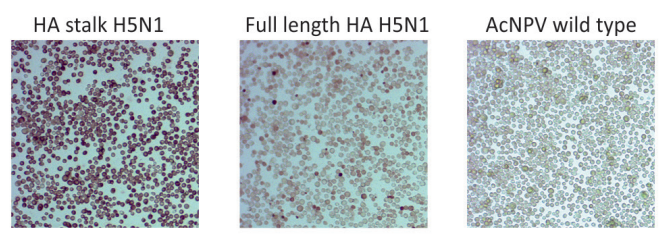

C

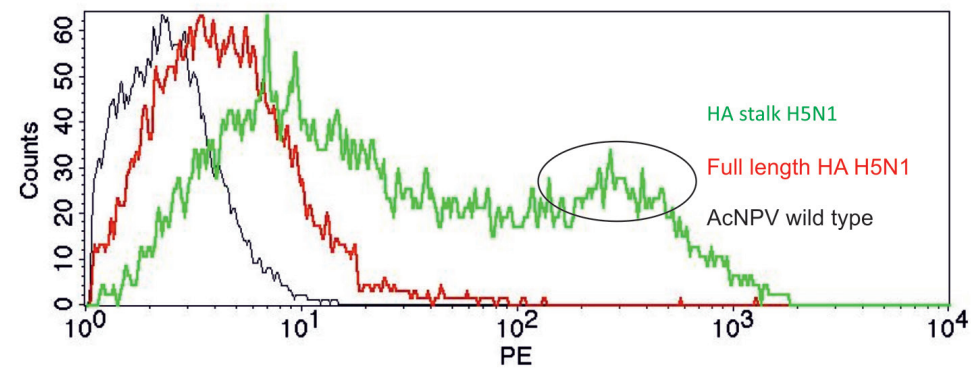

Figure 4. Example of analysis of HA stalk H5N1 recombinant protein reactivity with different antibodies (anti-H1N1 MAbs) in Western blotting (A), IPMA (B) and FACS (C).

(A) Western blotting analysis of cytosolic and membrane fractions containing HA stalk recombinant protein with H1N1 MAbs. Sf9 cells were infected with recombinant baculovirus HA stalk with m.o.i. $=1$ and collected after $48 \mathrm{~h}$ post infection. Cells were lysed and soluble and insoluble fractions were separated under reducing and non-reducing conditions. Western blotting was performed in order to examine the reactivity with H1N1 antibodies (1:1000 Sino Biological Inc.). (B) Cells were seeded in a 12-well culture plate and infected with recombinant baculovirus HA stalk with m.o.i. $=1$. After 48h IPMA test was performed using anti-H1N1 MAbs (1:1000 Sino Biological Inc.). (C) Cells were seeded in a 6-well culture plate and infected with recombinant baculovirus HA stalk with m.o.i. = 1. After $48 \mathrm{~h}$ cell surface expression of HA stalk recombinant proteins was determined using anti-H1N1 MAbs (1:1000 Sino Biological Inc.) by indirect immunofluorescence and analyzed using the FACS Calibur flow cytometer.

We observed that HA stalk recombinant protein reacted with polyclonal antibodies raised against influenza virus type A strain $\mathrm{H} 5 \mathrm{~N} 1$, against $\mathrm{HA} 2$ subunit of $\mathrm{H} 5 \mathrm{~N} 1$, against $\mathrm{H} 5 \mathrm{~N} 1$ reference strain, H5N2 VLA and $\mathrm{H} 5 \mathrm{Nx}$ chicken polyclonal antibody.

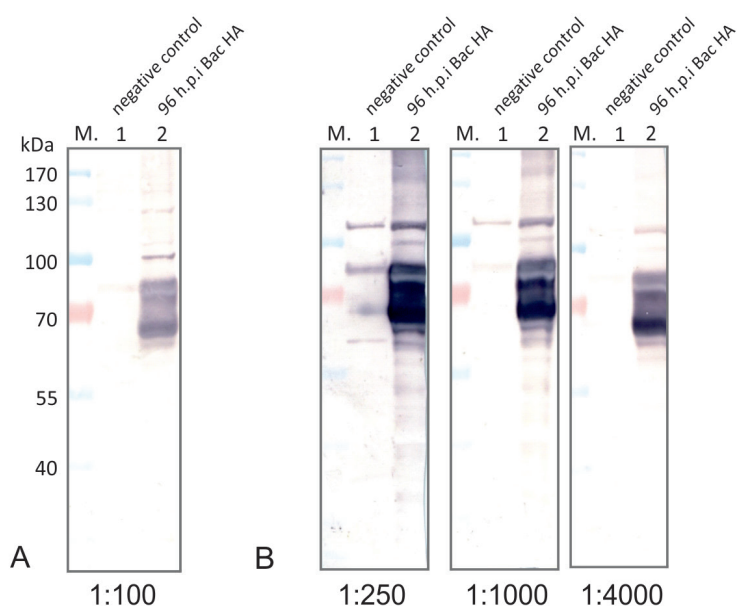

Figure 5. Western blot analysis of rHA H5N1 using polyclonal serum obtained after immunization of a rabbit with purified recombinant HAO protein.

A rabbit was immunized with recombinant HAO protein purified from PVDF membrane. First immunization was with $100 \mu \mathrm{g}$ of protein and the second after 3 weeks with $100 \mu \mathrm{g}$ of protein. (A) reference anti-H5N1 from VLA, (B) anti- rHA- H5N1; Western Blot analysis of the reactivity of obtained polyclonal serum with different recombinant antigens. Lane $\mathrm{M}$ - Protein Ladder, lane $1-$ negative control - WT ACNPV, lane $2-$ recombinant full length $\mathrm{HA}$ (70 kDa), membrane fraction after 96 h.p.i.
HA stalk was able to react with rabbit polyclonal as well as mouse monoclonal antibodies against H1N1 pandemic strain. In full length hemagglutinin, HA2 subunit is thought to be masked by the membrane-distant portion of HA1 subunit, the globular head domain. Thus, epitopes localized in the stalk domain were not recognized by anti H1N1 mAbs.

H5N1 HA stalk protein also reacted with antibodies against HAs from phylogenetic group 2 (Tables 2 and 3) which implies that the antigen described in this study contains the conserved, probably universal epitopes.

\section{Reactivity of HA stalk with universal human neutralizing antibody FI6}

FI6 antibody recognizes all subtypes of the influenza A virus hemagglutinin. As the FI6 antibody recognized only conformational epitopes we decided to investigate its reactivity with HA stalk antigen. In the IPMA test we examined whether recombinant $\mathrm{H} 5 \mathrm{~N} 1 \mathrm{HA}$ stalk protein reacts with FI6 human neutralizing antibody. FI6 specifically recognized HA stalk protein (Fig. 6).

\section{DISCUSSION}

Influenza virus causes 250000-500000 deaths worldwide annually. Potential global pandemic could kill millions (Kang et al., 2012). Current seasonal influenza vaccines are designed to provide strain-specific protection against two circulating subtypes of influenza A virus (H1N1 and $\mathrm{H} 3 \mathrm{~N} 2$ ) and one influenza B virus (Doyle et al., 2013). The rapid spreading of the 2009 pandemic $\mathrm{H} 1 \mathrm{~N} 1$ influenza virus was a signal that universal influenza vaccines are necessary, so that they would broadly protect against many mutated strains ( $\mathrm{Lu}$ et al., 2014). 


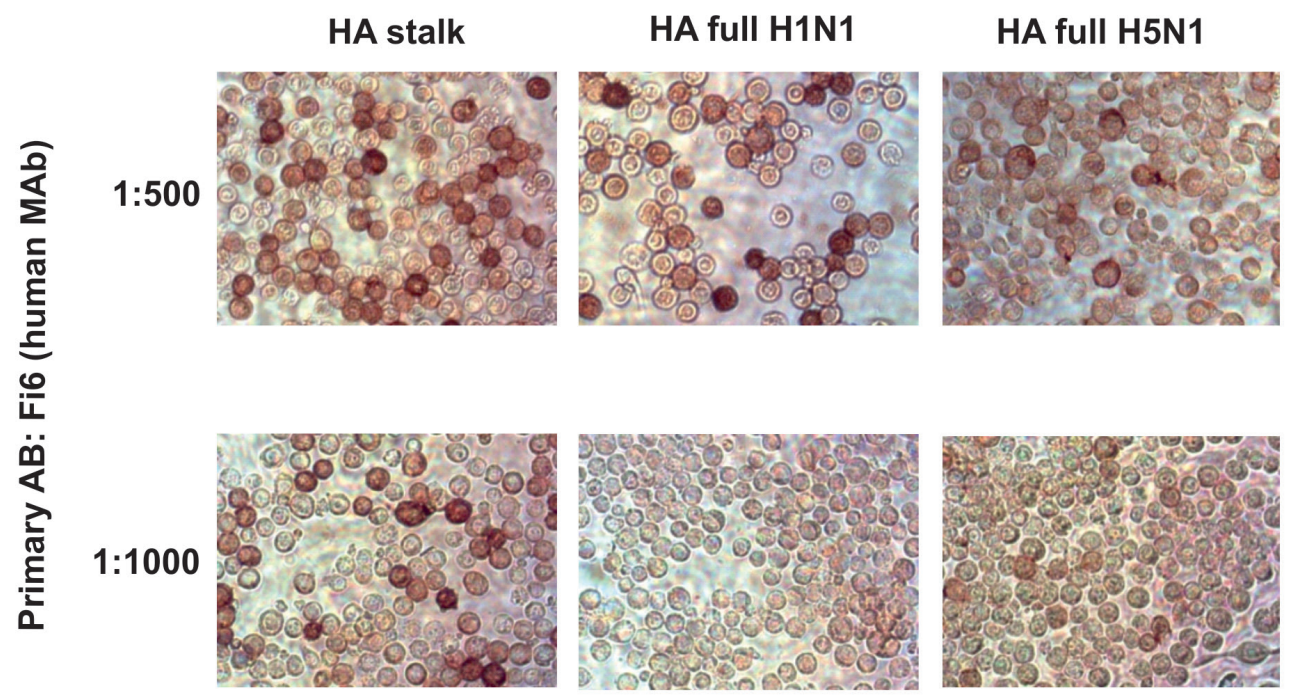

Figure 6. Analysis of immunoreactivity of H5N1 HA stalk recombinant protein with conformational, universal human FI6 MAb. Cells were seeded in a 12-well culture plate and infected with recombinant baculovirus HA stalk with m.o.i. = 1. After 48h IPMA test was performed using anti-FI6 MAbs dilutions 1:500,1:1000.

Current efforts for development of a universal influenza virus vaccine are mostly based on two strategies: the application of new approaches for delivery of existing antigens constructs (nanoplatforms) and the design of novel immunogens. Because of their great potential of cross-reactivity, HA stalk domain, the M2 protein and neuraminidase were identified as the universal antigenic targets (Pica \& Palese, 2013).

Recent studies describe the expression and characterization of HA stalk domain from human influenza viruses produced in mammalian cells (Steel et al., 2010; Krammer et al., 2012a). Based on current knowledge we decided to express the recombinant hemagglutinin stalk domain gene from H5N1 influenza A virus in the baculovirus system with the aim to determine its potential as a universal antigen for vaccine design. To produce homotrimeric "headless" HA in insect cells, we retained Cys 52 and Cys 277 (H3 numbering) (Steel et al., 2010).

Cleavage site of the headless HA of HPAI strain was recognized by insect cells proteases. We observed three major bands most likely representing three forms of the expressed recombinant protein: HA stalk composed of $\mathrm{N}$ - and C-terminal parts of HA1 subunit plus all of HA2 subunit, single HA2 subunit and $\mathrm{N}$ - and C-terminal parts of HA1 subunit linked by four glycines.

The universal HA stalk antibodies and "headless" HA protein from H1N1 strain obtained in mammalian cells are currently being studied. (Steel et al., 2010; Krammer et al., 2012a). Therefore, we planned to investigate whether the H5N1 "headless" construct produced in insect cells would also be recognized by different antibodies. We assumed that hemagglutinin of the avian viruses would probably contain conserved epitopes which were previously described for hemagglutinins of human Influenza viruses (Krammer et al., 2012b). Our results show that recombinant $\mathrm{H} 5 \mathrm{~N} 1 \mathrm{HA}$ stalk domain cross-reacts with various antibodies raised against group 1 and group 2 HAs, which confirms this hypothesis.

Previously, it has been shown that infection in humans with the pandemic swine influenza virus induces antibodies with specificity to the stalk domain of the viral hemagglutinin (Krammer et al., 2012a). In accordance with this result we observe the strongest immunoreactivity of pandemic H1N1 antibodies with the HA stalk do- main, which suggests that HA from H1N1 has a distinct structure that results in intensive production of antibodies that recognize conserved epitopes within HA stalk domain. We also planned to test whether immunization of rabbits with full length $\mathrm{H} 5 \mathrm{~N} 1$ hemagglutinin would elicit the anti-stalk antibodies. The results achieved demonstrate that obtained polyclonal antibodies reacts with recombinant hemagglutinin stalk domain protein, confirming that a pool of antibodies against HA stalk domain is raised after immunization with $\mathrm{H} 5 \mathrm{~N} 1$ influenza.

Our results showing that HA H5N1 vaccination elicits cross-reactive stem-directed antibodies were in accordance with those of Whittle JR and co-workers (Whittle et al., 2014).

In the last few years many research groups were involved in the identification and characterization of the universal antibodies that recognize different HAs (Corti et al., 2011). The HA stalk domain is responsible for fusion of viral and host membranes so that the virus can enter human cells. The FI6 antibody makes extensive contacts with conserved epitopes of the stalk, preventing viral entry ( $\mathrm{Lu}$ et al., 2014). We were curious whether the lack of globular head may have an impact on the efficacy of FI6 binding. In this report we show that exposure of HA stalk domain results in elevated immunoreactivity with FI6 as compared to full length HA (H5N1 as well as H1N1) (Fig. 6). Therefore, we suggest that in the absence of immunodominant globular head domain, the vaccination with HA stalk may elicit larger pool of antibodies against universal epitopes localized in the HA2 subunit.

Summary, there is an urgent need for formulation of universal vaccine against influenza virus. Many scientists investigate which antigen has the ability to give a broad rage protection. Our results confirm the presence of universal epitopes within the HA2 subunit in the stalk domain. It leads us to conclusion that with properly engineered HA stalk antigen we may obtain a potentially universal antigen.

\section{Acknowledgements of financial support}

This work was supported by Innovative Economy Program, Grant No. WND-POIG.01.01.02-00-007/08 in frame of Vaccine Cluster Consortium (VCC): Institute 
of Biochemistry and Biophysics, Polish Academy of Sciences, Warsaw, Poland (IBB); Institute of Biotechnology and Antibiotics, Warsaw, Poland (IBA); Department of Recombinant Vaccines, Intercollegiate Faculty of Biotechnology, University of Gdansk and Medical University of Gdansk, Gdansk, Poland (UG); Kucharczyk TE sp. z o.o. ,Warsaw, Poland (KTE); Institute of Animal Reproduction and Food Research, Polish Academy of Sciences, Tuwima 10, 10-747 Olsztyn, Poland (IAR); Department of Poultry Diseases, National Veterinary Research Institute, Pulawy, Poland (PIWet).

\section{REFRENCES}

Bouvier NM, Palese P (2008) The biology of influenza viruses. Vaccine 26 (Suppl 4): D49-D53.

Corti D, Suguitan AL Jr, Pinna D, Silacci C, Fernandez-Rodriguez BM, Vanzetta F, Santos C, Luke CJ, Torres-Velez FJ, Temperton NJ, Weiss RA, Sallusto F, Subbarao K, Lanzavecchia A (2010) Heterosubtypic neutralizing antibodies are produced by individuals immunized with a seasonal influenza vaccine. I Clin Invest 120: 1663-1673.

Corti D, Voss J, Gamblin SJ, Codoni G, Macagno A, Jarrossay D, Vachieri SG, Pinna D, Minola A, Vanzetta F, Silacci C, Fernandez-Rodriguez BM, Agatic G, Bianchi S, Giacchetto-Sasselli I, Calder L, Sallusto F, Collins P, Haire LF, Temperton N, Langedijk JP, Skehel JJ, Lanzavecchia A (2011) A neutralizing antibody selected from plasma cells that binds to group 1 and group 2 Influenza A hemagglutinins. Science 333: 850856.

Doyle TM, Hashem AM, Li C, Van Domselaar G, Larocque L, Wang J, Smith D, Cyr T, Farnsworth A, He R, Hurt AC, Brown EG, Li $\mathrm{X}$ (2013) Universal anti-neuraminidase antibody inhibiting all influenza A subtypes. Antiviral Res 100: 567-574.

Gamblin SJ, Skehel JJ (2010) Influenza Hemagglutinin and Neuraminidase Membrane Glycoproteins. I Biol Chem 285: 28403-28409.

Gromadzka B, Smietanka K, Dragun J, Minta Z, Gora-Sochacka A, Szewczyk B (2008) Detection of changes in avian influenza genome fragments by multitemperature single strand conformational polymorphism. Molecular and Cellular Probes 22: 301-304.

Hopkins R, Esposito D (2009) A rapid method for titrating baculovirus stocks using the Sf-9 Easy Titer cell line. Biotechniques 47: 785-788.

Kang SM, Kim MC, Compans RW (2012) Virus-like particles as universal influenza vaccines. Expert Rev V accines 11: 995-1007.

Kang SM, Song JM, Compans RW (2011) Novel vaccines against influenza viruses. Virus Research 162: 31-38.

Krammer F, Margine I, Tan GS, Pica N, Krause JC, Palese P (2012a) A carboxy-terminal trimerization domain stabilizes conformational epitopes on the stalk domain of soluble recombinant hemagglutinin substrates. PLOS One 7: e43603.
Krammer F, Pica N, Hai R, Tan GS, Palese P (2012b) Hemagglutinin stalk-reactive antibodies are boosted following sequential infection with seasonal and pandemic H1N1 Influenza virus in mice. J Virol 86: 10302-10307.

Lu Y, Welsh JP, Swartz JR (2014) Production and stabilization of the trimeric influenza hemagglutinin stem domain for potentially broadly protective influenza vaccines. Proc Natl Acad Sci USA 111: $125-130$.

Margine I, Hai R, Albrecht RA, Obermoser G, Harrod AC, Banchereau J, Palucka K, García-Sastre A, Palese P, Treanor JJ, Krammer F (2013a) H3N2 influenza virus infection induces broadly reactive hemagglutinin stalk antibodies in humans and mice. J Virol 87: 4728-4737.

Margine I, Krammer F, Hai R, Heaton NS, Tan GS, Andrews SA, Runstadler JA, Wilson PC, Albrecht RA, García-Sastre A, Palese P (2013b) Hemagglutinin stalk-based universal vaccine constructs protect against group 2 Influenza A viruses. J Virol 87: 10435-10446.

Pica N, Hai R, Krammer F, Wang TT, Maamary J, Eggink D, Tan GS Krause JC, Moran T, Stein CR, Banach D, Wrammert J, Belshe RB, García-Sastre A, Palese P (2012) Hemagglutinin stalk antibodies elicited by the 2009 pandemic influenza virus as a mechanism for the extinction of seasonal H1N1 viruses. Natl Acad Sci USA 109: 2573-2578.

Pica N, Palese P (2013) Toward a universal influenza virus vaccine: prospects and challenges. Annu Rev Med 64: 189-202.

Sangster MY, Baer J, Santiago FW, Fitzgerald T, Ilyushina NA, Sundararajan A, Henn AD, Krammer F, Yang H, Luke CJ, Zand MS, Wright PF, Treanor JJ, Topham DJ, Subbarao K (2013) B cell response and hemagglutinin stalk-reactive antibody production in different age cohorts following $2009 \mathrm{H} 1 \mathrm{~N} 1$ influenza virus vaccination. Clin Vaccine Immunol 20: 867-876.

Steel J, Lowen AC, Wang TT, Yondola M, Gao Q, Haye K, GarcíaSastre A, Palese P (2010) Influenza virus vaccine based on the conserved hemagglutinin stalk domain. MBio 1: 1-9.

Steinhauer DA (1999) Role of hemagglutinin cleavage for the pathogenicity of influenza virus. Virology 258: 1-20.

Whittle JR, Wheatley AK, Wu L, Lingwood D, Kanekiyo M, Ma SS, Narpala SR, Yassine HM, Frank GM, Yewdell JW, Ledgerwood JE, Wei CJ, McDermott AB, Graham BS, Koup RA, Nabel GJ (2014) Flow cytometry reveals that $\mathrm{H} 5 \mathrm{~N} 1$ vaccination elicits cross-reactive stem-directed antibodies from multiple Ig heavy chain lineages. J Virol 88: 4047-4057.

Wrammert J, Koutsonanos D, Gui-Mei Li, Edupuganti S, Sui J, Morrissey M, McCausland M, Skountzou I, Hornig M, Lipkin WI, Mehta A, Razavi B, Del Rio C, Zheng NY, Lee JH, Huang M, Ali Z, Kaur K, Andrews S, Amara RR, Wang Y, Das SR, O’Donnell CD, Yewdell JW, Subbarao K, Marasco WA, Mulligan MJ, Compans R, Ahmed R, Wilson PC (2011) Broadly cross-reactive antibodies dominate the human B cell response against 2009 pandemic H1N1 influenza virus infection. J Exp Med 208: 181-193. 